\title{
In Community Dwelling Females Age 65 or Older, Gait Speed Declines as Kinesthetic Awareness of the Lower Legs Decreases
}

\author{
Minsun Hong ${ }^{1}$, Ngozi Onyema ${ }^{2}$, Ayse Ozcan Edeer ${ }^{2 *}$, Valerie Olson ${ }^{3}$ \\ ${ }^{1}$ Kinderkamack Physical Therapy PC, Oradell, NJ, USA \\ ${ }^{2}$ Dominican College Doctor of Physical Therapy Program, Orangeburg, NY, USA \\ ${ }^{3}$ Messiah College Doctor of Physical Therapy Program, Mechanicsburg, PA, USA \\ Email: *ayse.edeer@dc.edu
}

How to cite this paper: Hong, M., Onyema, N., Edeer, A.O. and Olson, V. (2017) In Community Dwelling Females Age 65 or Older, Gait Speed Declines as Kinesthetic Awareness of the Lower Legs Decreases. $A d$ vances in Aging Research, 6, 17-28.

http://dx.doi.org/10.4236/aar.2017.61003

Received: November 3, 2016

Accepted: January 8, 2017

Published: January 11, 2017

Copyright (C) 2017 by authors and Scientific Research Publishing Inc. This work is licensed under the Creative Commons Attribution International License (CC BY 4.0).

http://creativecommons.org/licenses/by/4.0/

\begin{abstract}
Purpose: This study investigates the existence of a correlation between the gait speed and kinesthetic sense in lower legs and to conduct further analysis of kinesthetic sense in relation to the risk of fall among community dwelling females aged 65 years or older. Materials and Methods: A non-experimental correlational, descriptive, and cohort study included 38 community dwelling females (average age of 82.5 years). The kinesthetic ruler (K-Ruler) and kinesthetic test protocol were created and used to assess lower extremity kinesthetic awareness. The GaitRite System was used to assess gait speed. Each subject was categorized into four groups: "LL" (Low K-Score and low gait speed), "LH" (Low K-Score and high gait speed), "HL" (High K-Score and low gait speed), and "HH" (High K-Score and high gait speed) according to kinesthetic awareness and gait speed measured. Voluntary fall incidence reporting over the 6-month period was followed by the initial data collection. Results: Pearson product-moment correlation (2-tailed) showed that there is a statistically significant, positive moderate-to-strong correlation between $\mathrm{K}$ score and gait speed (ICC $=0.692, \mathrm{p}<0.05$ ). Additionally there were statistically significant negative moderate to strong correlations between age and $\mathrm{K}$ score, and age and gait speed (ICC $=-0.648$, and -0.596 respectively, $\mathrm{p}<$ 0.05). The 13 subjects reported fall incidences over the 6-month period. No falls were reported among subjects in HL group. Discussion: Gait speed declines as kinesthetic awareness of lower extremity decreases in community dwelling 65 or older female subjects. Additionally, the combination of kinesthetic awareness and gait speed can be served as a predictor of fall risk. The $\mathrm{K}$-ruler can be used to assess lower extremity kinesthetic awareness in older people as a feasible and standard test.
\end{abstract}




\section{Keywords}

Gait Speed, Kinesthesia, Risk of Falls, Elderly Female

\section{Introduction}

The decline of sensory and motor functions with aging often affects the static or dynamic postural control, which may lead to a limitation of functional ambulation [1]. Kinesthesia and proprioception are the major factors affecting the property of gait. The decline of kinesthetic sense leads to an increase in the risk of falls among the elderly population [2]. According to Center of Disease Control \& Prevention Factsheet, 1 in 3 elderly women age 65 or older have fall injuries [3]. Alamgir et al. (2012) quotes, "Fall injury is a leading cause of death and disability among older adults" [4].

Many studies have been conducted to assess gait speed as a predictor of the risk of future disability among the elderly population and evidenced that the lower the gait speed the greater the risk of falls and future problems [5] [6] [7] [8] [9]. Slower gait can be interpreted as a measure of how much the energetic cost of walking increased due to the decline of executive or sensorimotor functions, such as muscle strength, balance, proprioception, kinesthesia with age [10]. Quach et al. (2012) interpreted slower gaits as an adoptive mechanism to prevent slips, trips, and falls, therefore elderly people with slower gaits have low risk of fall [11].

Kinesthetic awareness, by definition, is awareness of the direction and magnitude of the extremity or joint movement. Decreased kinesthetic sense leads to increased risk of falls in older people. However, devices to measure kinesthesia are very rare. Mostly custom designed kinesthetic measuring devices have been used to assess upper extremity and trunk position sense in a healthy population. This explains why there are no prior studies that have assessed the relationship between kinesthesia and gait speed and kinesthesia and falls. A study by Mishra et al. (2013) is the only study we could find that mentioned any relationship between kinesthesia and falls stating that the decline of kinesthetic sense leads to an increase in the risk of falls among the elderly population [2].

We thought that the kinesthetic awareness would be one of the predictors of the risk of fall in older people beside the gait speed changes. However, no standard kinesthetic testing protocol or equipment was currently available to measure lower extremity kinesthetic awareness. This required the researcher to create a new kinesthetic testing device and protocol to assess kinesthetic awareness in elderly people based on the description of kinesthesia test in the book of O'Sullivan Schmitz, and Fulk [12].

Based on the literature review, the researcher aimed to use kinesthetic awareness tool to assess the kinesthetic sense of lower extremity in older people as one of the risks of fall. The primary focus of the study was to investigate linear correlation between the kinesthetic awareness of the lower legs and the gait speed 
and to make further analysis of the study results in relation to the risk of falls among the community dwelling female population age 65 or older. Our hypothesis was "In community dwelling females age 65 or over, the gait speed declines as kinesthetic awareness of the lower legs decreases."

\section{Materials and Methods}

A non-probability sampling technique of convenience sampling was used for a non-experimental research method with a descriptive, correlational, and cohort design. This employed a single snapshot data collection of general health information, two kinesthetic tests of each leg in a chair sitting position, and a gait speed at a normal pace with or without an assistive device on a level gait platform via GaitRite System. For the cohort study, we recorded subjects' voluntary reporting of their fall incidences at the end of each month for the 6-month period followed by the initial data collection.

\subsection{Subjects}

The information sessions of our study were held with a community dwelling elderly females age 65 or older living at Convents in Blauvelt and Sparkill, NJ and an independent living facility in Nanuet after approval by the Dominican College Institutional Review Board (IRB) project (IRB\# 2015-0308-010). Initially, the total 41 subjects were recruited on a voluntary basis at information sessions held in the three locations. Two subjects voluntarily dropped out on the data on collection day and one subject was excluded according to the study exclusion criteria. Therefore, data collection was made for the final 38 subjects who participated in the study.

\subsection{Inclusion and Exclusion Criteria}

Our inclusion criteria is a community dwelling female aged 65 or older. Our exclusion criteria are abnormal vital signs, oxygen saturation rate $\left(\mathrm{SpO}_{2}\right)<90 \%$, partial or non-weight bearing restrictions, ambulation that requires more than contact guard assistance (CGA), lower extremity (LE) muscle strength less than $3 / 5$, knee range of motion (ROM) less than functional ROM, or anybody who did not have an authorization from the primary physician or nurse practitioner to participate in the study. We also excluded anyone who had a Mini Mental State Exam [13] score less than 24, who was considered cognitively impaired [14].

\subsection{Procedure}

The entire data collection procedure took about 20 minutes for each participant, which included a brief screening (mini mental state test, vital signs and oxygen saturation rate), two kinesthetic awareness tests, and the gait speed measurement. Confidentiality was maintained during the entire data collection. During the health screening, we obtained the subject's weight, height, leg length, knee/ leg pain, if the pain affected their gait speed, any use of assistive devices (AD), or 
other health related issues that may affect our study. Seven subjects reported that their knee/leg pain affected their gait speeds. Twelve subjects were using $\mathrm{AD}$ during ambulation.

\subsection{Kinesthetic Test}

The two kinesthetic tests consist of Procedure-1 and Procedure-2, which each participant requires to perform 10 trials for each leg after two test trials. Both procedures are performed in a seated position on a chair with eyes blinded to block the subject's visual sense in order to enhance the accurate assessment of the subject's kinesthetic awareness.

Procedure- 1 assesses the ability to detect the direction of a subtle movement of the test leg. The test leg was placed on a skateboard and the subject asked to make a simultaneous verbal response of "forward or backward" as the examiner moved the test leg passively by moving the skate board forward or backward to avoid the subject's tactile stimulation. O'Sullivan et al. (2014) states, "the therapist's grip should remain minimal to reduce tactile stimulation" [12]. The subject receives a score "1" for an accurate and timely response (within 1 second); otherwise, a score of " 0 ". Total K-Score (sum of all scores) for Procedure-1 ranges from " 0 " to "20" (0 to 10 for each leg) as shown in Table 1.

Procedure- 2 assesses the ability to coordinate the kinesthetic senses. K-Ruler (Figure 1) placed on the floor such that both feet (with shoes off) land right above K-Ruler to avoid friction during the movement of feet. K-Ruler is specifically designed to measure the active and passive foot displacements of both feet. The tip of the second toe on each leg is the point of reference used to read the scale on K-Ruler as the legs move as shown in Figure 1. The subject is asked to spontaneously move the test leg matching to the direction and the magnitude of

Table 1. Procedure 1 and 2 scoring system.

\begin{tabular}{|c|c|c|c|c|c|}
\hline $\begin{array}{c}\text { Procedure } 1 \\
\text { Passive movement } \\
\text { trials }\end{array}$ & Right leg & Left leg & $\begin{array}{c}\text { Procedure } 2 \\
\text { Active movement } \\
\text { trials }\end{array}$ & Right leg & Left leg \\
\hline 1. Forward & & & Trial 1-Scale 1 & & \\
\hline 2. Forward & & & Trial 2-Scale 2 & & \\
\hline 3. Backward & & & Trial 3-Scale-1 & & \\
\hline 4. Backward & & & Trial 4-Scale-2 & & \\
\hline 5. Backward & & & Trial 5-Scale 3 & & \\
\hline 6. Forward & & & Trial 6-Scale 5 & & \\
\hline 7. Backward & & & Trial 7-Scale 1 & & \\
\hline 8. Forward & & & Trial 8-Scale-1 & & \\
\hline 9. Forward & & & Trial 9-Scale-4 & & \\
\hline 10. Backward & & & Trial 10-Scale 7 & & \\
\hline Total Score & & & Total Score & & \\
\hline
\end{tabular}



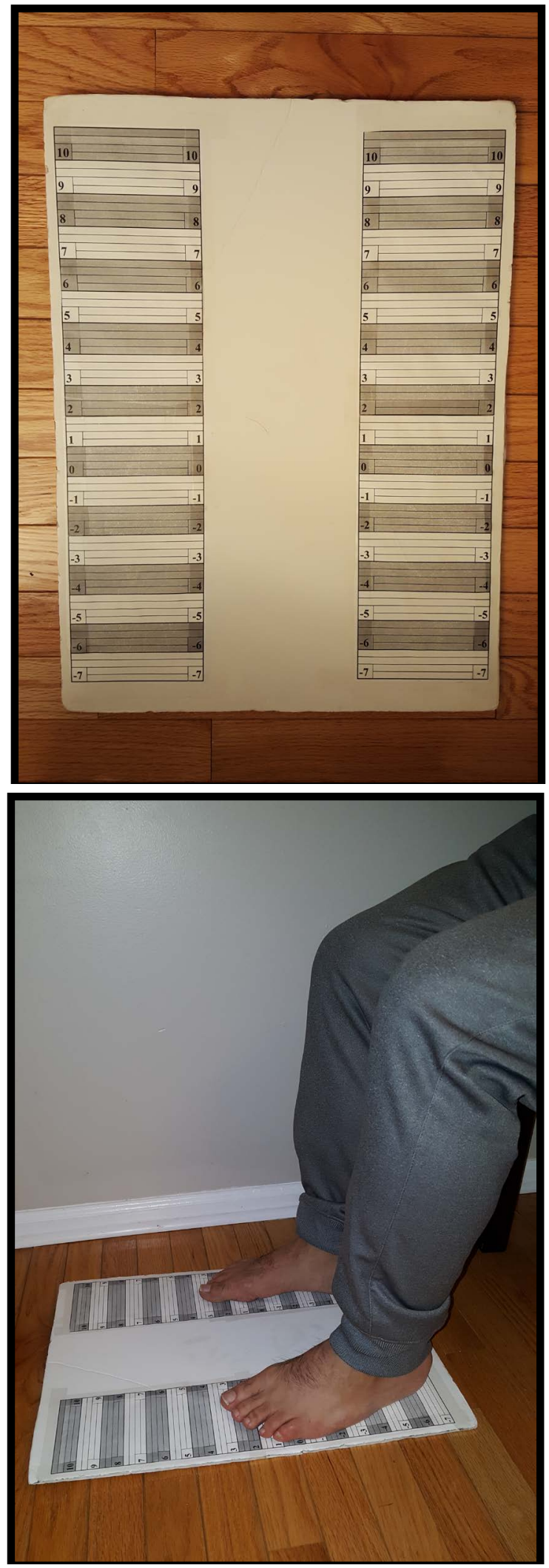

Figure 1. Kinesthetic Ruler designed to save time and efforts in the multiple measurements of the test or nontest legs movements during the kinesthetic awareness test-Procedure 2. 
the passive movement of non-test leg made by the examiner. The subject receives "0" point in the following cases: 1 ) the subject's test leg moves totally off in the direction, 2) the magnitude is off more than $3 / 4$ inches from the exact scale of the non-test leg movement, or 3) it takes more than 2 seconds to match the movement. The subject receives " 2 " points if the subject matched test leg simultaneously to the direction and the magnitude of the non-test leg movement within the range within $1 / 4$ inches. The subject receives " 1 " point for all other cases. Total K-Score for Procedure-2 ranges 0 and 40 ( 0 to 20 for each leg) as shown in Table 1.

The total K-Score that summed for Procedure 1 and 2 ranges 0 to 60 ( 0 to 30 for each leg) for each subject, where K-Score " 0 " represents the total impairment of kinesthesia for both legs and "60" represents kinesthesia intact for both legs. For the data analysis, we used the total K-score as a representation of each subject's kinesthetic awareness.

\subsection{Gait Analysis}

We used gait analysis system (GaitRite Electronic Walkway, manufactured by CIR Systems Inc) with a gait platform with multiple sensors that provides the temporal and spatial information of the subject's gait. Subjects started to walk about 20 feet before the GaitRite platform in order to get their normal pace of walk before they reached the platform and continued walking at a normal pace about 20 feet on the level GaitRite platform. Each subject had two trials, with or without $\mathrm{AD}$, and was provided the required level of assistance as documented during the health screening interview. Gait speed for each trial was recorded and averaged. The average gait speed of the two trials was used for our data analysis.

\subsection{Fall Incidence Report}

Each subject reported voluntarily the number of fall incidents they experienced each month for the 6-month period following the initial data collection. A fall is defined as an unintentional rest on the ground or other lower level. Also, if a subject had to grab an object in order not to fall at the time when the subject had an unintentional loss of balance, it is considered as a fall.

\subsection{Statistical Analysis}

All statistical analyses were conducted on IBM Statistical Package for the Social Sciences (SPSS) Version 18.0. Central tendency and variability of the participants age, K-score, and gait speed were used to describe the sample population. The relationship between the gait speed and K-Score, and additionally age and $\mathrm{K}$-score and age and gait speed were investigated using the two-tailed Pearson Product-Moment Correlation Coefficient (PPMCC). The alpha level for significance was $\mathrm{p}=0.05$ and confidence interval (CI) was set at $95 \%$.

\section{Results}

The average age, K-score, and gait speed of the 31 participants were 83.4 years 
$(\mathrm{SD}=7.8), 39.6$ points $(\mathrm{SD}=5.8)$, and $68.6 \mathrm{~cm} / \mathrm{sec}(\mathrm{SD}=24.4)$, respectively. Pearson product-moment correlation (2-tailed) showed that there is a statistically significant positive moderate to strong correlation between K-score and gait speed $(\mathrm{ICC}=0.692, \mathrm{p}<0.05)$. Additionally there were statistically significant negative moderate to strong correlations between age and $\mathrm{K}$-score and age and gait speed (ICC $=-0.648$, and -0.596 , respectively, $\mathrm{p}<0.05$ ). Figures $2-4$ show the corresponding graphs.

During data analysis, we found 7 outliers identified with K-Score and gait speeds outside the data range of most subjects. In our study we excluded the 7

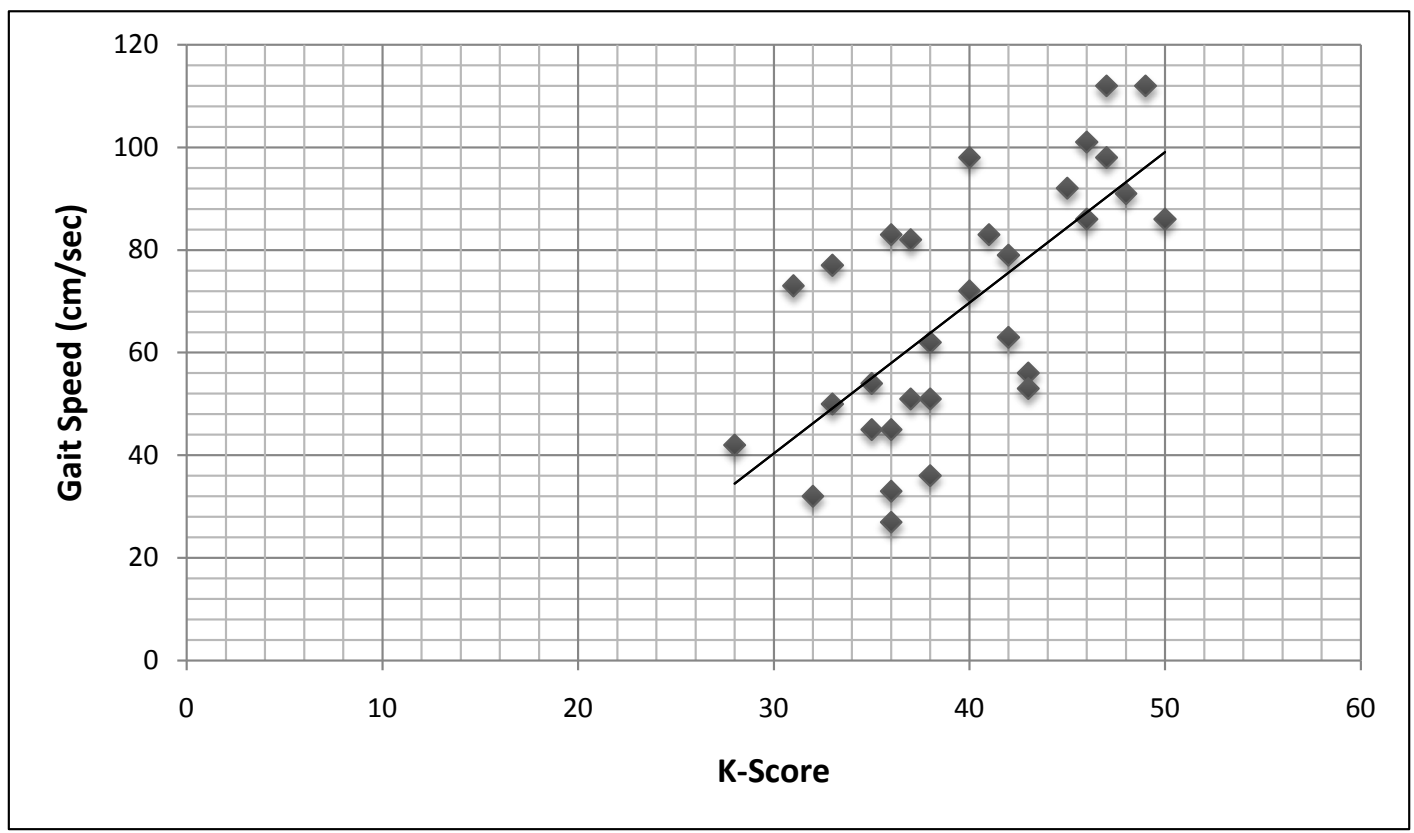

Figure 2. Correlation between K-score and adjusted-velocity $(\mathrm{ICC}=0.692, \mathrm{p}<0.05)$.

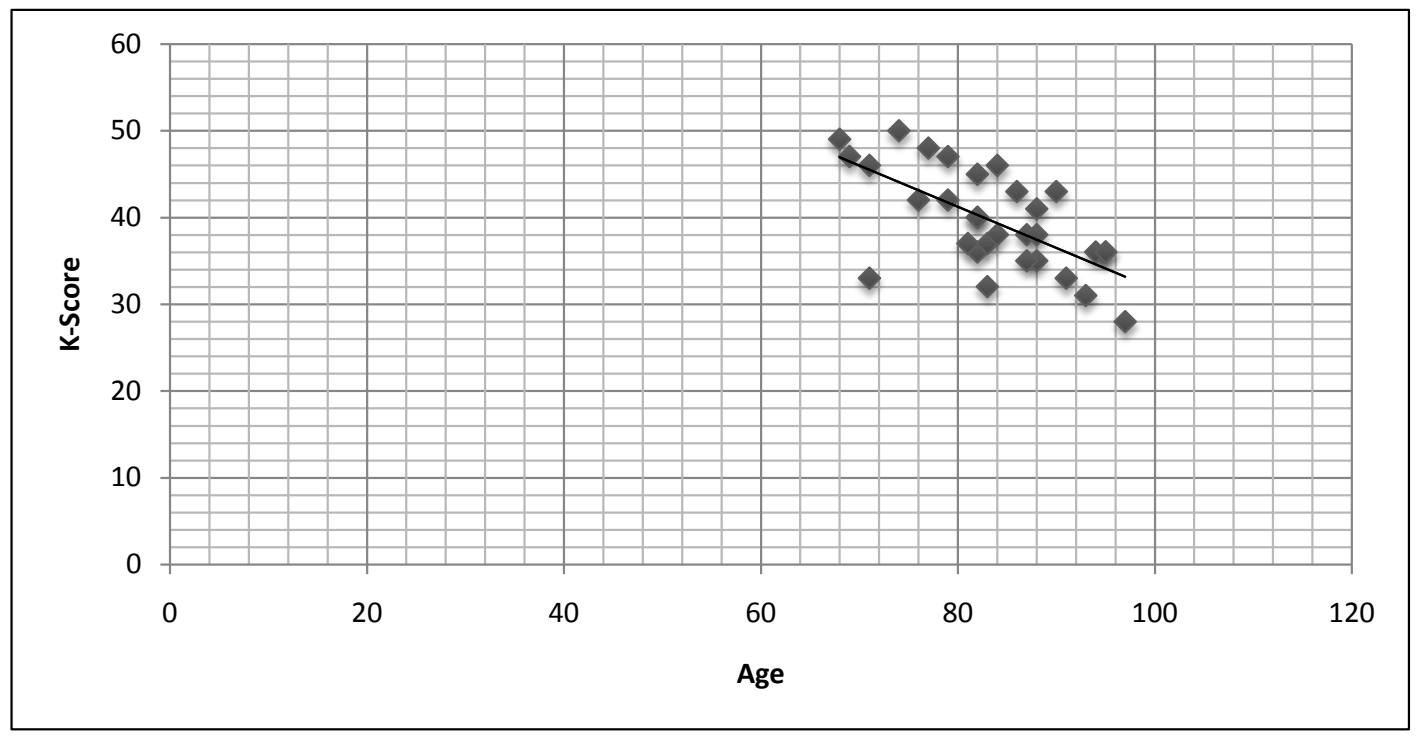

Figure 3. Correlation between age and K-score (ICC $=-0.648, \mathrm{p}<0.05)$. (ICC $=-0.596$, respectively, $\mathrm{p}<$ $0.05)$. 


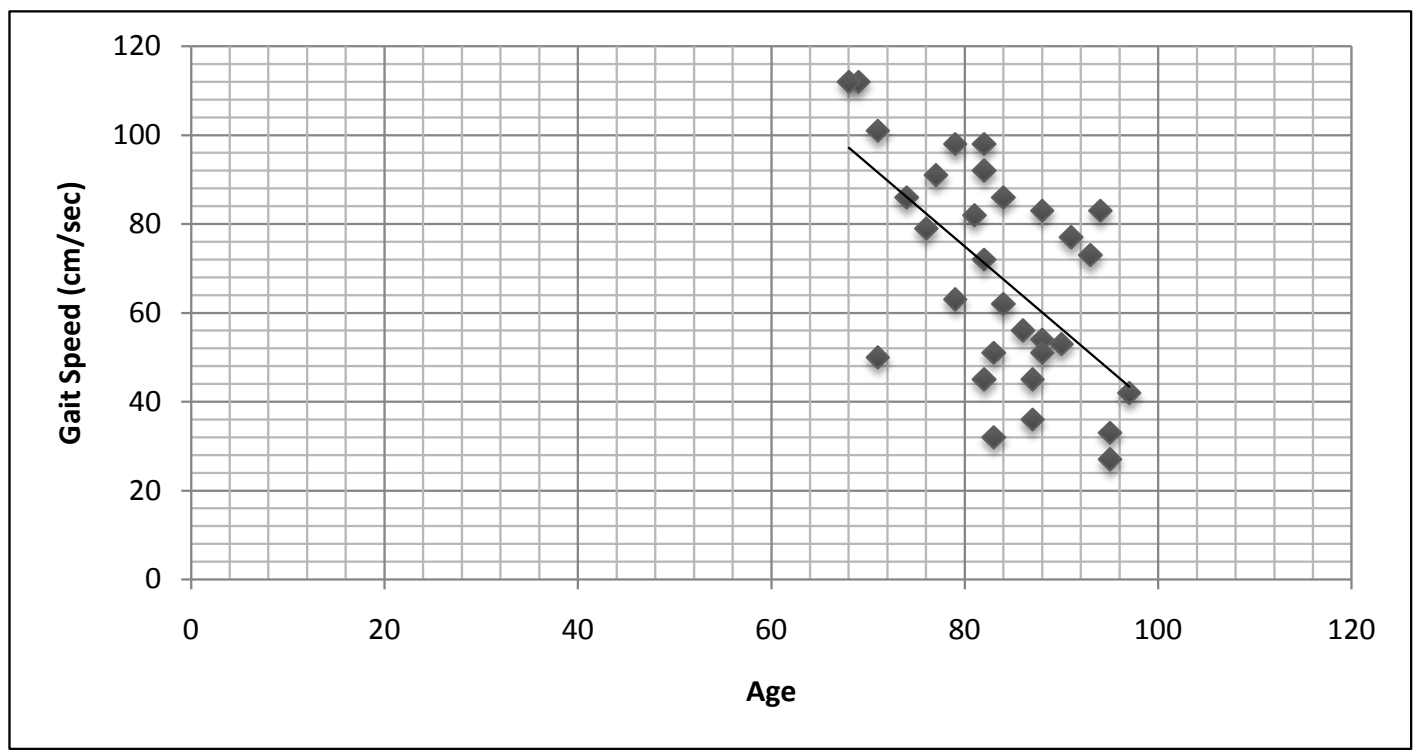

Figure 4. Correlation between age and gait speed (ICC $=-0.596$, respectively, $\mathrm{p}<0.05)$.

outliers to avoid the potential bias in the inferential statistic analysis of the sample population.

Studies have shown that increased BMI are correlated with decreased gait speed [15] [16] [17] [18]. We carefully examined possible impacts of BMI on gait speed and developed the BMI adjustment factor using BMI data we collected among the 38 subjects. Each subject's gait speed has been calibrated reflecting the subject's BMI adjustment factors of 0.7 for BMI $<20$ and 1.1 for BMI $>25$ (Table 2).

For the purpose of cohort study of fall incidences, we divided the 38 subjects into 4 subgroups:

1) Low Low (LL): subjects with both K-Score and gait speeds below its average,

2) Low High (LH): subjects with K-Score below its average, gait speed above its average,

3) High Low (HL): subjects with K-Score above its average and gait speed below its average,

4) High High ( $\mathrm{HH})$ : subjects with both K-Score and gait speed above its average.

There were 13 out of 38 subjects (34\%) who reported their fall incidences over the 6-month period. Five out of 15 subjects (or 33\%) in LL-group, 4 out of 7 subjects (or 57\%) in LH-group, 0 (zero) out of 4 subjects (or 0\%) in HL-group, and 4 out of 12 subjects (or 33\%) for HH-group. Interestingly, the $57 \%$ of all subjects in LH-group(low K-score with high gait speed) experienced falls, while $0 \%$ (zero) of all subject in HL-group (high K-score with slow gait) experienced no falls. Figure 5 shows comparison of fall incidences by subgroup.

\section{Discussion}

In many studies the gait speed alone is viewed as a predictor of risk of falls. 
Table 2. Body Mass Index (BMI) adjustment factors for each age band.

\begin{tabular}{cccccccc}
\hline BMI & Count & $\begin{array}{c}\text { Average } \\
\text { age }\end{array}$ & $\begin{array}{c}\text { Average } \\
\text { BMI }\end{array}$ & $\begin{array}{c}\text { Average } \\
\text { K-score }\end{array}$ & $\begin{array}{c}(2) \\
\text { Average } \\
\text { gait speed } \\
\text { at each } \\
\text { BMI band }\end{array}$ & $\begin{array}{c}\text { Average } \\
\text { gait speed } \\
\text { at each } \\
\text { age band }\end{array}$ & $\begin{array}{c}\text { BMI } \\
\text { Adjustment } \\
\text { Factor }= \\
(2) /(1)\end{array}$ \\
\hline Under 20 & 6 & 87.5 & 18 & 38.0 & 82 & 54 & 0.7 \\
$20-24$ & 12 & 83.7 & 22 & 40.3 & 71 & 71 & 1.0 \\
$25-29$ & 8 & 83.5 & 28 & 34.6 & 64 & 71 & 1.1 \\
30 and over & 12 & 78.1 & 33 & 39.0 & 72 & 79 & 1.1 \\
Total & $\mathbf{3 8}$ & $\mathbf{8 2 . 5}$ & $\mathbf{2 6}$ & $\mathbf{3 8 . 4}$ & $\mathbf{7 1}$ & NA & NA \\
\hline
\end{tabular}

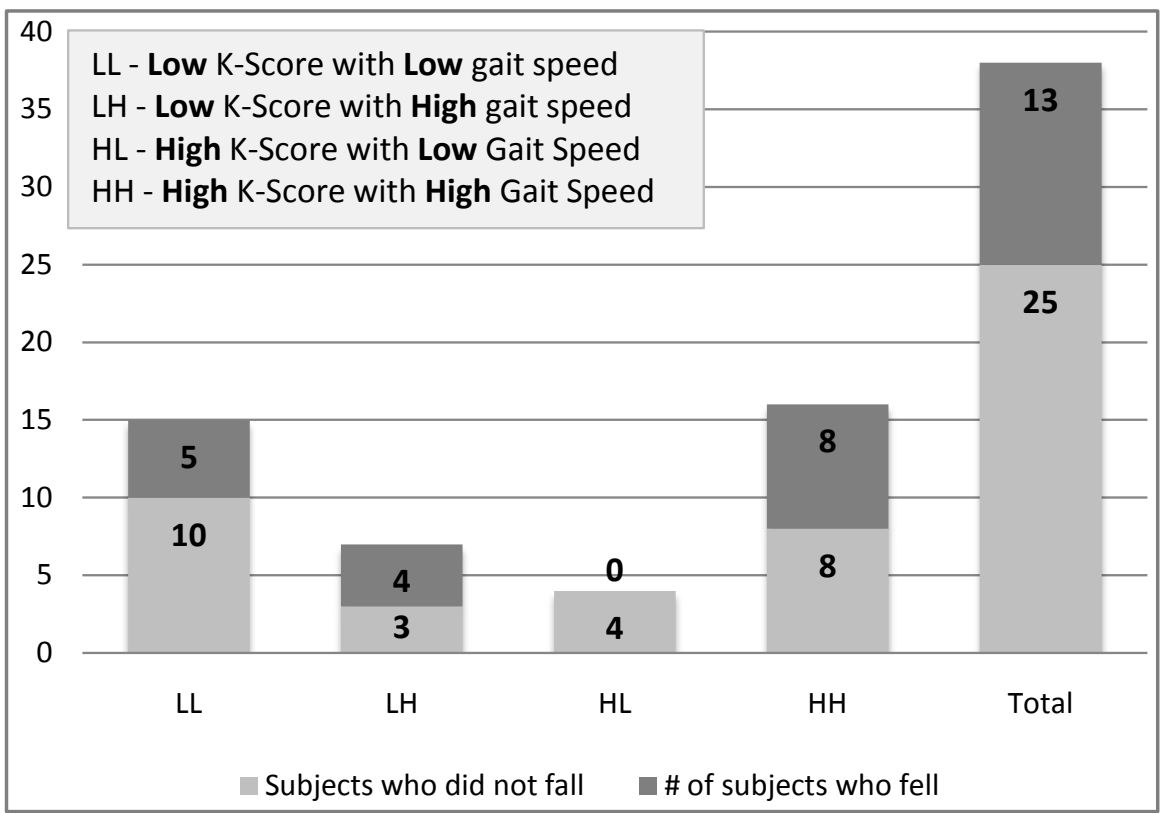

Figure 5. The number of subjects who fell over the six month period following the data collection date by Group.

Numerous studies proved that kinesthetic awareness as well as gait speed declines with aging [1] [2] [5] [6] [7] [8]. Our study results confirmed that both kinesthetic awareness and gait speed are negatively correlated with age. Also, the study results showed a positive correlation (ICC $=0.692, \mathrm{p}<0.5$ ) between kinesthetic awareness and the gait speed among elderly females.

In our study the GaitRite system was used to assess gait speed while other studies measured gait speed on a watch or chronometer [5] [6] [7] [8]. The GaitRite system has shown to be a valid tool for measuring spatial and temporal gait parameters in both healthy subjects and in subjects with abnormal gait patterns [19]. It is an automated system which allows for the measurement of gait speed without the worry of personal error as one might have measuring with, for example, a stop watch.

Many studies suggested a link between a risk of falls and gait speed in elderly people. One study found that consciously slowing one's gait speed might help 
decrease the risk of falls [20]. Another study found that slower gait speeds were associated with greater risk of indoor falls and faster gait speeds were associated with greater risk of outdoor falls [11]. In the study of Mishra et al. (2013) kinesthesia may affect the property of gait and its decline with aging may lead to increase in risk of falls [2].

In our cohort study we did not find a link between fall risk and kinesthetic awareness or gait speed alone. However we divided the 38 subjects into 4 subgroups for the purpos of cohort study of fall incidences. Our study showed that subjects in HL-group (higher kinesthetic awareness and low gait speed) had zero falls, while, 57\% of LH-group (lower kinesthetic awareness with faster gait speed) experienced falls and 33\% of subjects in LL and HH groups experienced falls. The study results suggested that elderly females with relatively lower kinesthetic awareness and relatively higher gait speed have the highest risk of falls. Elderly females with relatively higher kinesthetic awareness and relatively lower gait speed have the lowest risk of falls. Based on these findings, we can argue that high kinesthesia and slow gait speed suggests a lower risk of fall, and low kinesthesia and fast gait speed indicates a higher risk of fall.

Kinesthetic sensitivity is not easily assessed in older people because of their impaired cognitive abilities. The testing must be easily understood by the older people, simple to administer and should produce objective measures for subsequent assessments. With these requirements in mind, a K-ruler and test protocol were designed to assess the lower extremity kinesthetic awareness. We conclude that the K-ruler is a feasible, user-friendly, and standard test to assess kinesthetic awareness in older females. Therefore it can be a part of the fall risk assessment in older people to minimize the risk of fall.

Further studies are warranted to investigate the effect of kinesthetic training on the kinesthetic ability and fall risk. Yet, we cannot generalize our study results due to the study limitations even if the study results signified the existence of a positive correlation between kinesthetic awareness and gait speed. The study limitations are the potential bias of convenience sampling, relatively small sample size $(\mathrm{N}=38)$, the lack of verification of psychometric properties of K-ruler used as kinesthetic awareness test tool.

\section{Conclusion}

Based on our study results, we concluded that a positive correlation exists between kinesthetic awareness and gait speed. Additionally we also concluded that the combination of the kinesthetic sense and gait speed could serve as a predictor of a risk of fall among the elderly female population. Our research study is the first study to assess lower extremity kinesthetic awareness for the risk of falls and to investigate its relationship with gait speed in elderly females. Although the validity and reliability of the K-ruler have not been searched, we believe that it can be used to assess kinesthetic awareness in older people to as a vital part of the fall risk assessment. Furthermore, kinesthetic training should be investigated as a preventive care in relation to the risk of falling and to be utilized to prevent 
elderly people from catastrophic falls.

\section{References}

[1] Rogers, M.W., Tamulevicius, N., Semple, S.J. and Krkeljas, Z. (2012) Efficacy of Home-Based Kinesthesia, Balance \& Agility Exercise Training among Persons with Symptomatic Knee Osteoarthritis. Journal of Sports Science and Medicine, 11, 751-758.

[2] Mishra, P., Sharma, A., Bhat, L. and Narwhal, R. (2013) Analyzing Age Related Changes in Proprioceptive and Kinesthetic Sensations in Community Dwelling Elderly Subjects. Asian Journal of Multidisciplinary Studies, 1, 1-8.

[3] Centers for Disease Control and Prevention, Important Fact about Falls, Last Updated on January 20, 2016.

http://www.cdc.gov/homeandrecreationalsafety/falls/adultfalls.html

[4] Alamgir, H., Muazzam, S. and Nasrullah, M. (2012) Unintentional Falls Mortality among Elderly in the United States: Time for Action. Injury, 43, 2065-2071. https://doi.org/10.1016/j.injury.2011.12.001

[5] Montero-Odasso, M., Schapira, M., Soriano, E.R., Varela, M., Kaplan, R., et al. (2005) Gait Velocity as a Single Predictor of Adverse Events in Healthy Seniors Aged 75 Years and Older. Journals of Gerontology Series A: Biological Sciences and Medical Sciences, 60A, 1304-1309. https://doi.org/10.1093/gerona/60.10.1304

[6] Luukinen, H., Koski, K., Laippala, P. and Kivelä, S.L. (1995) Predictors for Recurrent Falls among the Home-Dwelling Elderly. Scandinavian Journal of Primary Health Care, 13, 294-299. https://doi.org/10.3109/02813439508996778

[7] Chu, L.W., Chi, I. and Chiu, A.Y. (2005) Incidence and Predictors of Falls in the Chinese Elderly. Annals of the Academy of Medicine, Singapore Journal, 34, 60-72.

[8] Dargent-Molina, P., Favier, F., Grandjean, H., Baudoin, C., Schott, A., Hausherr, E., et al. (1996) Fall-Related Factors and Risk of Hip Fractures: The EPIDOS Prospective Study. Lancet, 348, 145-149. https://doi.org/10.1016/S0140-6736(96)01440-7

[9] Brach, J.S., Perera, S., Van Swearingen, J.M., Hile, E.S., Wert, D.M., et al. (2011) Challenging Gait Conditions Predict 1-Year Decline in Gait Speed in Older Adults with Apparently Normal Gait. Physical Therapy Journal, 91, 1857-1864. https://doi.org/10.2522/ptj.20100387

[10] Schrack, J.A., Simonsick, E.M., Chaves, P.H and Ferrucci, L. (2012) The Role of Energetic Cost in the Age-Related Slowing of Gait Speed. Journal of the American Geriatrics Society, 60, 1811-1816. https://doi.org/10.1111/j.1532-5415.2012.04153.x

[11] Quach, L., Galica, A.M., Jones, R.N., Procter-Gray, E., Manor, B., Hannan, M.T., et al. (2011) The Nonlinear Relationship between Gait Speed and Falls: The Maintenance of Balance, Independent Living, Intellect, and Zest in the Elderly of Boston Study. Journal of the American Geriatrics Society, 59, 1069-1073. https://doi.org/10.1111/j.1532-5415.2011.03408.x

[12] O’Sullivan, S.B., Schmitz, T.J. and Fulk, G.D. (2014) Physical Rehabilitation. 6th Edition, FA Davis Company, Philadelphia, 108-109.

[13] Kurlowicz, L. and Wallace, M. (1999) The Mini-Mental State Examination (MMSE). Journal of Gerontological Nursing, 25, 8-9. https://doi.org/10.3928/0098-9134-19990501-08

[14] Hartford Institute for Geriatric Nursing. Mini Mental State Examination. Division of Nursing, New York University, New York. www.hartfordign.org

[15] Tomohiro, D., Shin-ichi, D., Masanobu, U. and Hiroki, S. (2014) Examination of Factors Affecting Gait Properties in Healthy Older Adults: Focusing on Knee Ex- 
tension Strength, Visual Acuity, and Knee Joint Pain. Journal of Geriatric Physical Therapy, 37, 52-57. https://doi.org/10.1519/JPT.0b013e318295daba

[16] Watson, N., Rosano, C., Boudreau, R., Simonsick, E., Ferrucci, L., et al. (2010) Executive Function, Memory, and Gait Speed Decline in Well-Functioning Older Adults. Journals of Gerontology Series A: Biological Sciences and Medical Sciences, 65A, 1093-1100. https://doi.org/10.1093/gerona/glq111

[17] Hergenroeder, A.L., Wert, D.M., Hile, E.S., Studenski, S.A. and Brach, J.S. (2011) Association of Body Mass Index with Self-Report and Performance-Based Measures of Balance and Mobility. Physical Therapy, 91, 1223-1234. https://doi.org/10.2522/ptj.20100214

[18] Kitayuguchi, J., Kamada, M., Hamano, T., Nabika, T., Shiwaku, K., et al. (2016) Association between Knee Pain and Gait Speed Decline in Rural Japanese Community-Dwelling Older Adults: 1-Year Prospective Cohort Study. Geriatrics \& Gerontology International, 16, 55-64. https://doi.org/10.1111/ggi.12432

[19] Webster, K.E., Wittwer, J.E. and Feller, J.A. (2005) Validity of the GAITRite Walkway System for the Measurement of Averaged and Individual Step Parameters of Gait. Gait \& Posture, 22, 317-321. https://doi.org/10.1016/j.gaitpost.2004.10.005

[20] Kelsey, J.L., Prill, M.M., Keegan, T.M., Tanner, H.E., Bernstein, A.L., Queensberry, C.J., et al. (2005) Reducing the Risk for Distal Forearm Fracture: Preserve Bone Mass, Slow Down, and Don't Fall! Osteoporosis International, 16, 681-690. https://doi.org/10.1007/s00198-004-1745-8

\section{Abbreviations}

CGA: Contact Guard Assistance,

LE: Lower Extremity,

ROM: Range of Motion,

AD: Assistive Device,

SD: Standard Deviation,

BMI: Body Mass Index,

ICC: Interclass Correlation Coefficient,

LL: Low Low,

LH: Low High,

HL: High Low,

$\mathrm{HH}$ : High High. 
Submit or recommend next manuscript to SCIRP and we will provide best service for you:

Accepting pre-submission inquiries through Email, Facebook, LinkedIn, Twitter, etc. A wide selection of journals (inclusive of 9 subjects, more than 200 journals)

Providing 24-hour high-quality service

User-friendly online submission system

Fair and swift peer-review system

Efficient typesetting and proofreading procedure

Display of the result of downloads and visits, as well as the number of cited articles Maximum dissemination of your research work

Submit your manuscript at: http://papersubmission.scirp.org/

Or contact aar@scirp.org 